\title{
Robust Feature Extraction and Iris Recognition for Biometric Personal Identification
}

\author{
Rahib Hidayat Abiyev and Kemal Ihsan Kilic \\ Department of Computer Engineering, Near East University, Nicosia, \\ North Cyprus
}

\section{Introduction}

Humans have distinctive and unique traits which can be used to distinguish them from other humans, acting as a form of identification. A number of traits characterising physiological or behavioral characteristics of human can be used for biometric identification. Basic physiological characteristics are face, facial thermograms, fingerprint, iris, retina, hand geometry, odour/scent. Voice, signature, typing rhythm, gait are related to behavioral characteristics. The critical attributes of these characteristics for reliably recognition are the variations of selected characteristic across the human population, uniqueness of these characteristics for each individual, their immutability over time (Jain et al.,1998). Human iris is the best characteristic when we consider these attributes.

The texture of iris is complex, unique, and very stable throughout life. Iris patterns have a high degree of randomness in their structure. This is what makes them unique. The iris is a protected internal organ and it can be used as an identity document or a password offering a very high degree of identity assurance. Also the human iris is immutable over time. From one year of age until death, the patterns of the iris are relatively constant (Jain et al.,1998, Adler,1965). Because of uniqueness and immutability, iris recognition is one of accurate and reliable human identification technique.

Nowadays biometrics technology plays important role in public security and information security domains. Iris recognition is one of the most reliable and accurate biometrics that plays an important role in identification of individuals. The iris recognition method deliver accurate results under varied environmental circumstances. Iris is the part between the pupil and the white sclera. The iris texture provides many minute characteristics such as freckles, coronas, stripes, furrows, crypts (Adler,1965). These visible characteristics are unique for each subject.

Iris recognition process can be separated into these basic stages: iris capturing, preprocessing and recognition of the iris region. Each of these steps uses different algorithms. Pre-processing includes iris localization, normalization, and enhancement. In iris localization step, the detection of the inner (pupillary) and outer (limbic) circles of the iris and the detection of the upper and lower bound of the eyelids are performed. The inner circle is located on the iris and pupil boundary, the outer circle is located on the sclera and iris boundary. Today researchers follow different methods in finding pupillary and limbic 
boundaries. For these methods, the important problems are the accuracy of localization of iris boundaries, preprocessing speed, robustness (Daugman, 1994, Noh et al.,2003). For the iris localization step, several techniques have been developed. Daugman used integrodifferential operator to detect inner and outer boundaries of the iris (Daugman, 2001; Daugman, 2003; Daugman, 2006). First derivative of image intensity and Hough transform methods are proposed for localization of iris boundaries in (Wields, 1995). Using edge detection, the segmentation of iris is performed in (Boles \& Boashash, 1998). Circular Hough transform is applied to find the iris boundaries in (Masek,2003). In (Ma et al., 2002) greylevel information, Canny edge detection and Hough transform are applied for iris segmentation. In these researches iris locating algorithm based on the grey distribution of the features is presented. (Tisse et al., 2002) used a method that can locate a circle given three non-linear points. This is used for finding relatively accurate location of the circle, then the gradient decompose of Hough transform is applied for the accurate location of pupil. (Kanag \& Xu, 2000) used Sobel operator and applied circle detection operator to locate the pupil boundary. In (Yuan et al., 2005) using threshold value, Canny operator and Hough transform the inner circle of iris is determined, then edge detection operator is used for outer circle. Pupil has dark colour, but in certain non ideal conditions because of specular reflections it can be illuminated unevenly. For such irregular iris images, researchers used intensity based techniques for iris localization (Daugman, 2007; Vatsa et al.,2008). (Vatsa et al.,2008) used two stage iris segmentation algorithm. In the first stage, iris boundaries are estimated by using elliptical model. In the second stage Mumford-Shah function is used to detect exact boundaries. Authors of (Zuo et al.,2008) explained robust automatic segmentation algorithm. One of the most important characteristics of iris localization system is its processing speed. Sometimes for the iris localization it may not be possible to utilize any method involving floating-point processing. For example if we have small embedded real-time system without any floating-point processing part, operations involving kernel convolutions will be unusable, even if they may offer more accurate results. Detailed discussions on the issues related to performance of segmentation methods can be found in (Liu etal.,2005, Cui et al,2004, Abiyev \& Altunkaya,2008,2009). Iris localization generally takes nearly more than half of the total processing time in the recognition systems, as pointed in (Cui et al,2004). With this vision, we develop fast segmentation algorithms that require not many floating-point processing like convolution.

After the preprocessing stage feature vectors of iris images are extracted. Theses feature vectors are used for recognition of irises. Image recognition step basically deals with the "meaning" of the feature vectors, in which classification, labelling, matching occur. Various algorithms have been applied for feature extraction and pattern matching processes. These methods use local and global features of the iris.

Among many methods used for recognition today, these can be listed: Phase based approach (Daugman,2001; Dougman,2003; Dougman \& Downing,1994, Miyazawa et al.,2008), wavelet transform, zero crossing approach (Boles \& Boashash,1998; S.Avila \& S.Reillo, 2002; Noh et al.,2002; Mallat,1992), and texture analysis (Wields,1997; Boles \& Boashash,1998; Ma et al.,2003; Park et al.,2003; Ma et al.,2005). (Wang \& Han, 2003,2005) proposed independent component analysis is for iris recognition.

In phase based approach, the task of iris feature extraction is a process of phase demodulation. The phase is chosen as an iris feature. Daugman used multiscale quadrature wavelets to extract texture phase structure information of the iris to generate a 2,048-bit iris code and compared the difference between a pair of iris representations by 
computing their Hamming distance. Boles and Boashash (1998) calculated a zero-crossing representation of $1 \mathrm{D}$ wavelet transform at various resolution levels of a concentric circle on an iris image to characterize the texture of the iris. Zero-crossings of wavelet transform provide meaningful information of images structure (Mallat,1992). S.Avila and S.Reillo (2002) further developed the method of Boles and Boashash by using different distance measures for matching. Many texture analysis methods can be adapted to recognize the iris images (Wildes,1997; Ma et al.,2003). Wildes represented the iris texture with a Laplacian pyramid constructed with four different resolution levels and used the normalized correlation to determine whether the input image and the model image are from the same class. (Ma et al., 2002) used multichannel Gabor filters to extract iris features. Future bank of circular symmetric filters was designed to capture the discriminating information along the angular direction of the iris image (Ma et al., 2003). (Tisse et al., 2002) uses a directional filter bank in order to decompose the iris images into eight directional subband outputs. In (Ma et al.,2005), iris recognition algorithm based on characterizing local variations of image structures was utilized. In (Lim et al.,2001) moment based iris blob matching algorithm is proposed and combination of this algorithm with local feature based classifiers is considered. (Abiyev \& Altuknka,2008; Scotti, 2007) applied Neural Networks (NN) for iris pattern recognition.

Analysis of previous research demonstrated that the efficiency of personal identification system using iris recognition is determined by the fast and accurate recognition of the iris patterns. Great portion of the recognition time is spent on the localization of iris boundaries. Accuracy of recognition depends on the accuracy of iris localization and on the accuracy of classification. Some methods for iris localization are based on selecting threshold value for detecting pupil boundaries. Experiments on the CASIA Version 1 and Version 3 iris databases showed to us that segmentation algorithms sometimes may require intervention from researchers, for example fixing a certain threshold value for all the images. But this does not give good results, since many iris images captured under different illumination conditions and some of them contain noises. For example CASIA Version 1 and CASIA Version 3 databases have different illumination properties and CASIA Version 3 database iris images contain specular highlights in the pupil region. Existence of specular highlight poses difficulties for any algorithm that assumes pupil pixels have form of disc with the darkest pixels in the central region of the image. In this study we summarized our efforts on developing robust feature extraction methods. Two methods are proposed for feature extraction. The algorithms can find the image specific segmentation parameters from iris images. The first method is simple and fast method developed for high quality iris images (CASIA Version 1) where there is no specular highlight. Here black rectangle is used for pupillary boundary detection and gradient search method for limbic boundary detection. The second method is adaptive to degraded quality and can also perform iris segmentation in the presence of specular highlight (CASIA Version 3). Here Hough circle search for pupillary boundary detection and gradient search method for limbic boundary detection. After segmenttion the features are extracted and used for recognition of irises.With the development of NN, iris recognition systems may gain speed, accuracy, learning ability. In this paper fast iris localization algorithm and NN based iris classification are proposed.

The rest of the paper is organized as follows: Section 2 includes the structure of iris recognition system, iris localization, normalization and enhancement algorithms. Section 3 is about iris classification using neural network. Section 4 presents experimental results of implemented algorithms. Section 5 includes conclusions. 


\section{Image processing}

\subsection{Structure of the iris recognition system}

The block diagram of the iris recognition system is given in Fig.1. The image recognition system includes iris image acquisition and iris recognition. The iris image acquisition includes the lighting system, the positioning system, and the physical capture system (Wields,1997). The iris recognition includes preprocessing and neural networks. During iris acquisition, the iris image in the input sequence must be clear and sharp. Clarity of the iris's minute characteristics and sharpness of the boundary between the pupil and the iris, and the boundary between the iris and the sclera affects the quality of the iris image. A high quality image must be selected for iris recognition. In iris pre-processing, the iris is detected and extracted from an eye image and normalized. Normalized image after enhancement is represented by the feature vector that describes gray-scale values of the iris image. For classification neural network is used. Feature vector becomes the training data set for the neural network. The iris recognition system includes two operation modes: training mode and online mode. At fist stage, the training of recognition system is carried out using greyscale values of iris images. After training, in online mode, neural network performs classification and recognizes the patterns that belong to a certain person's iris.

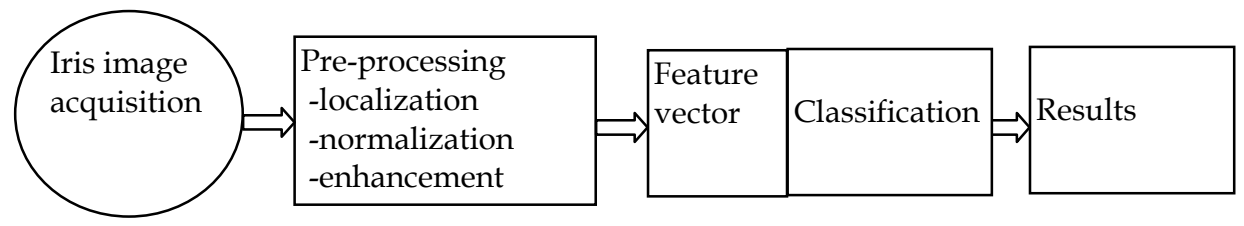

Fig. 1. A block diagram of the iris recognition system.

\subsection{Iris localization}

An eye image contains not only the iris region but also some parts that need to be separated from iris, such as the pupil, eyelids, sclera. For this reason, at the first step, segmentation should be done to localize and extract the iris region from the eye image. Iris localization is the detection of the iris area between pupil and sclera. So we need to detect the upper and lower boundaries of the iris and determine its inner and outer circles (Fig.2). Two different algorithms for localization of iris patterns are presented here. The first algorithm is called black-rectangle algorithm that uses grey values for segmentation, second algorithm uses Otsu thresholding and Hough circle search for pupillary boundary detection and gradient search method for limbic boundary detection.

A number of algorithms has been developed for iris localization. Most of them are based on the Hough transform (Wields,1997; Masek,2003). In these researches the canny edge detection algorithm with circular Hough transform is applied to detect the inner and outer boundaries of the iris. The circular Hough transform is employed to reveal the radius and centre coordinates of the pupil and iris regions. In this operation, starting from the upper left corner of iris, the circular Hough transform is applied. This algorithm is used for each inner and outer circle separately. The application of the Hough transform requires a long time to locate the boundaries of the iris and need fixing a certain threshold value for all the images. But this does not give good results, since many iris images captured under different 
illumination conditions and some of them contain noise. In the paper two algorithms are proposed for iris localization. The first method is simple and fast method developed for high quality iris images (CASIA Version 1) where there is no specular highlight. Here black rectangle is used for pupillary boundary detection and gradient search method for limbic boundary detection. The second method is adaptive to degraded quality and can also perform iris segmentation in the presence of specular highlight (CASIA Version 3). Here Hough circle search is applied for pupillary boundary detection and gradient search method for limbic boundary detection. After segmentation the features are extracted and used for recognition of irises.

Black-rectangle algorithm. As mentioned iris localization includes detecting the boundaries between pupil and iris and also sclera and iris. To find the boundary between the pupil and iris, we must detect the location (centre coordinates and radius) of the pupil. The black rectangular technique that is applied in order to localize pupil and detect the inner circle of iris is given in algorithm 1. The pupil is a dark circular area in an eye image. Besides the pupil, eyelids and eyelashes are also characterized by black colour. In some cases, the pupil is not located in the middle of an eye image, and this causes difficulties in finding the exact location of the pupil using point-by- point comparison on the base of threshold technique. In this paper, we are looking for the black rectangular region in an iris image (Fig. 3). Choosing the size of the black rectangular area is important, and this affects the accurate determination of the pupil's position. If we choose a small size, then this area can be found in the eyelash region. In this paper a $(10 x 10)$ rectangular area is used to accurately detect the location of the pupil (step 1 of algorithm 1). Searching starts from the vertical middle point of the iris image and continues to the right side of the image (see step 2 of algorithm 1). A threshold value is used to detect the black rectangular area. Starting from the middle vertical point of iris image, the greyscale value of each point is compared with the threshold value. As it is proven by many experiments, the greyscale values within the pupil are very small. So a threshold value can be easily chosen. If greyscale values in each point of the iris image are less than the threshold value, then the rectangular area will be detected. If this condition is not satisfactory for the selected position, then the search is continued from the next position. This process starts from the left side of the iris, and it continues until the end of the right side of the iris. In case the black rectangular area is not detected, the new position in the upper side of the vertical middle point of the image is selected and the search for the black rectangular area is resumed. If the black rectangular area is not found in the upper side of the eye image, then the search is continued in the down side of image. In Fig. 3(a), the searching points are shown by the lines. In Fig. 3(b), the black rectangular area is shown in white colour. After finding the black rectangular area, we start to detect the boundary of the pupil and iris. At first step, the points located in the boundary of pupil and iris, in horizontal direction, then the points in the vertical direction are detected (Fig. 4). In Fig. 4 the circle represents the pupil, and the rectangle that is inside the circle represents the rectangular black area. The border of the pupil and the iris has a much larger greyscale change value. Using a threshold value on the iris image, the algorithm detects the coordinates of the horizontal boundary points of $\left(\mathrm{x}_{1}, \mathrm{y}_{1}\right)$ and $\left(\mathrm{x}_{1}, \mathrm{y}_{2}\right)$, as shown in Fig. 4. The same procedure is applied to find the coordinates of the vertical boundary points $\left(\mathrm{x}_{3}, \mathrm{y}_{3}\right)$ and $\left(\mathrm{x}_{4}, \mathrm{y}_{3}\right)$. After finding the horizontal and vertical boundary points between the pupil and the iris, the following formula is used to find the centre coordinates $\left(x_{p}, y_{p}\right)$ of the pupil. 
Fig. 2. A localised iris image.

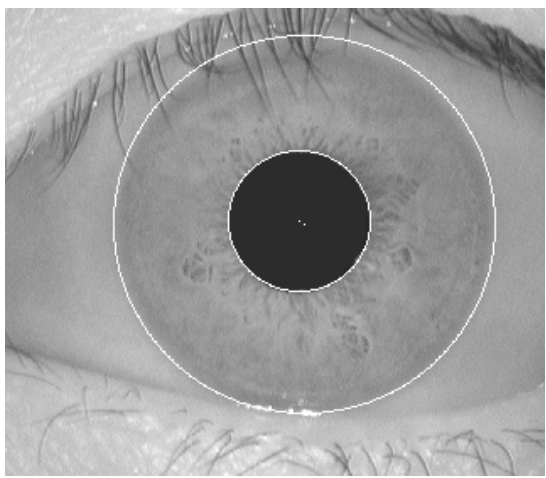

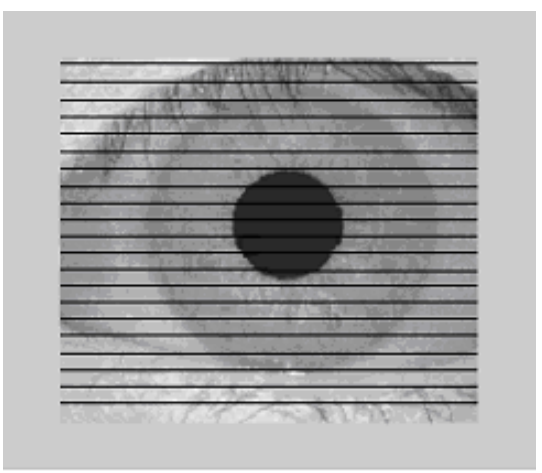

(a)

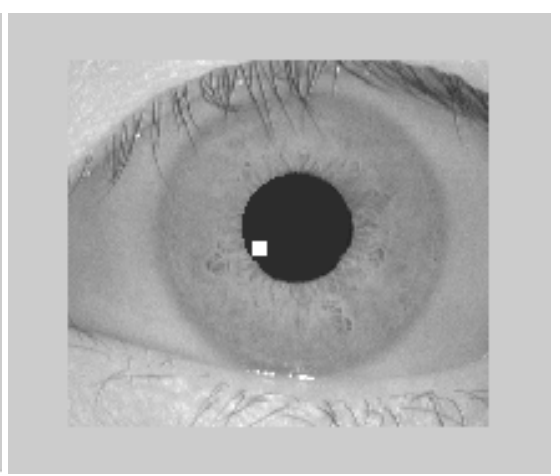

(b)

Fig. 3. Detecting the rectangular area: a) The lines that were drawn to detect rectangular areas, $b$ ) The result of detecting of rectangular area.

$$
x_{p}=\left(x_{3}+x_{4}\right) / 2, \quad \mathrm{y}_{\mathrm{p}}=\left(y_{3}+y_{4}\right) / 2
$$

\section{Algorithm 1}

1. Input of Iris image and Setting Size of Black Rectangular area

2. Determining Horizontal middle point of iris image and Searching Black Rectangular area in horizontal direction

3. If Black Rectangular is found

4. Then Detects the coordinates of the horizontal boundary points of $\left(x_{1}, y_{1}\right)$ and $\left(x_{1}, y_{2}\right)$, and vertical boundary points $\left(x_{3}, y_{3}\right)$ and $\left(x_{4}, y_{3}\right)$ between the pupil and the iris

5. Determining the centre coordinates $\left(x_{p}, y_{p}\right)$ of the pupil using equation 1.

6. Determining the radius of the pupil using equation (2).

7. Else Continue Searching in the down side (or in upper side) of image's middle point

8. Finding Outer boundary of pupil and Determining the differences using (3) in horizontal direction

9. Determining Maximum values of Diferences in left and right side of pupil and Finding boundary point between iris and sclera

10. Determining the centre coordinates $\left(\mathrm{x}_{\mathrm{s}}, \mathrm{y}_{\mathrm{s}}\right)$ and radius of the iris using equation 5 


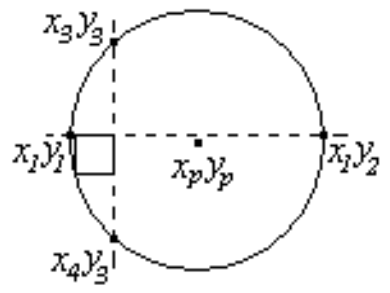

Fig. 4. Finding the centre of the pupil.

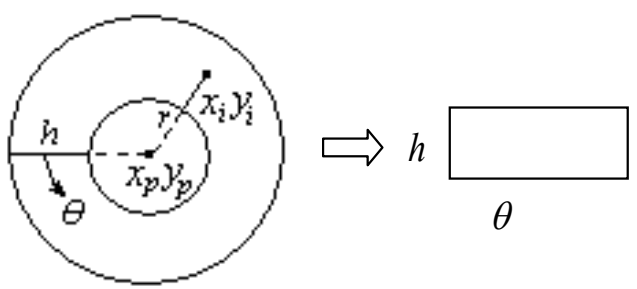

Fig. 5. Normalization of the iris.

The same procedure is applied for two different rectangular areas. In case of small differences between coordinates, the same procedure is applied for four and more different rectangular areas in order to detect a more accurate position of the pupil's centre. After determining the centre points, the radius of the pupil is computed using equation (2) (see steps 3-7 of algorithm 1).

$$
r_{p}=\sqrt{\left(x_{c}-x_{1}\right)^{2}+\left(y_{c}-y_{1}\right)^{2}}, \quad \text { or } \quad r_{p}=\sqrt{\left(x_{c}-x_{3}\right)^{2}+\left(y_{c}-y_{3}\right)^{2}}
$$

Because of the change of greyscale values in the outer boundaries of iris is very soft, the current edge detection methods are difficult to implement for detection of the outer boundaries. In this paper, another algorithm is applied in order to detect the outer boundaries of the iris. We start from the outer boundaries of the pupil and determine the difference of sum of greyscale values between the first ten elements and second ten elements in horizontal direction (see step 9 of algorithm 1). This process is continued in the left and right sectors of the iris. The difference corresponding to the maximum value is selected as boundary point. This procedure is implemented by the following formula.

$$
D L_{i}=\sum_{i=10}^{y_{p}-\left(r_{p}+10\right)}\left(S_{i+1}-S_{i}\right) ; \quad D R_{j}=\sum_{j=y_{p}+\left(r_{p}+10\right)}^{\text {right }-10}\left(S_{j+1}-S_{j}\right)
$$

Here $D L$ and $D R$ are the differences determined in the left and right sectors of the iris, correspondingly. $x_{p}$ and $y_{p}$ are centre coordinates of the pupil, $r_{p}$ is radius of the pupil, right is the right most $y$ coordinate of the iris image. In each point, $S$ is calculated as

$$
S_{j}=\sum_{k=j}^{k+10} I(i, k)
$$


where $i=x_{p}$, for the left sector of iris $j=10, \ldots, y_{p}-\left(r_{p}+10\right)$, and for the right sector of iris $j=y_{p}+\left(r_{p}+10\right) . I_{x}(i, k)$ are greyscale values.

The centre and radius of the iris are determined using

$$
y_{s}=(L+R) / 2, \quad r_{s}=(R-L) / 2
$$

$L=i$, where $i$ correspond to the value $\max \left(\left|D L_{i}\right|\right), R=j$, where $j$ correspond to the value $\max \left(\left|D R_{j}\right|\right.$ (see steps $10-11$ of algorithm 1 ).

Using inner and outer circles, the normalization of iris is performed (Fig.5).

Algorithm based on Otsu thresholding and Hough circle. Black-rectangle searching algorithm could be efficiently used for fast localization iris images of CASIA Version 1 database. When iris images contain specular highlights then the detection of pupil region is subjected to some difficulties. Black-rectangle searching algorithm is difficult to apply such iris images. In CASIA Version 3 database, iris images have specular highlights in the pupil region. These specular highlights pose difficulties for any algorithm that assumes pupil pixels having form of disc with the darkest pixels in the central region of the image. In such cases one useful method is the utilization of Hough transformation that searches for circles in the image in order to detect pupillary boundary. Experiences on the CASIA Version 1 and Version 3 iris databases showed to us that segmentation algorithms sometimes may require intervention from researchers, for example fixing a certain threshold value. But sometimes it is necessary to use an automatic algorithm that will decide on such parameters depending on the certain image characteristics (i.e. size, illumination, average gray level, etc...). In this algorithm the combination of thresholding and Hough circle method is used for detecting pupil boundaries. The steps of the algorithm can be seen in algorithm 2. Below, explanations on the motivations and reasons for the steps involved in the algorithm is given. Generally iris databases created under different illumination settings. For each one selecting a good threshold value by programmer is possible only after some experimentation.

Furthermore different images from the same database can be captured under different illumination settings. So finding an automatic thresholding method is essential to overcome such difficulties when thresholding is necessary to separate object (iris or pupil) and background. In the algorithm proposed in this paper, Otsu thresholding (Otsu,1979) is used for this purpose. Experiments with Otsu Thresholding have demonstrated that a thresholding process for detecting iris boundaries can be automated. In that way, instead of using programmer given threshold value, the process of extracting it from the image can be automated. Several experiments showed that half of the Otsu threshold value, perfectly fits for this purpose (Step 1-2 in algorithm 2). Fig. 6(a) shows sample eye image before any processing. The process of selecting Otsu threshold value is given in Fig. 6(b). Here right vertical line demonstrates Otsu threshold value, and the left vertical line demonstrates half of Otsu threshold value. In some cases of thresholding, Otsu value by itself separates sclera from the rest of the eye in the image. So taking half of the Otsu value is a good approximation (also good heuristics) for separating pupil from the sclera region. Here multilevel Otsu methods can be utilized for better thresholding, which can be computationally more expensive.

Interested readers can find good reviews and surveys on thresholding and segmentation methods in (Sezgin \& Sankur, 2004; Trier \& Taxt,1995]. Separating pupil region from the image can let us to find certain characteristic of the pupil, at least approximate radius size when the "pupil pixels" are counted. Of course any thresholding that can separate pupil will 


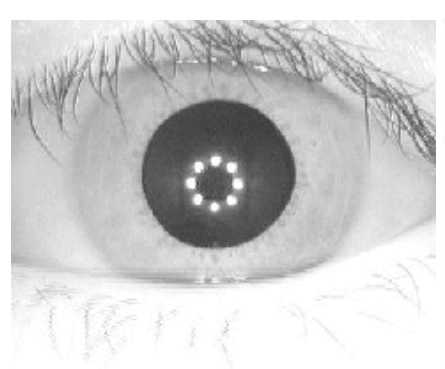

(a) Original Image

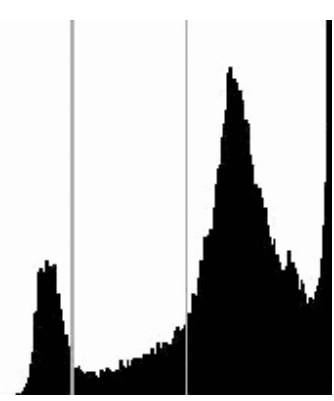

(b) its Histogram

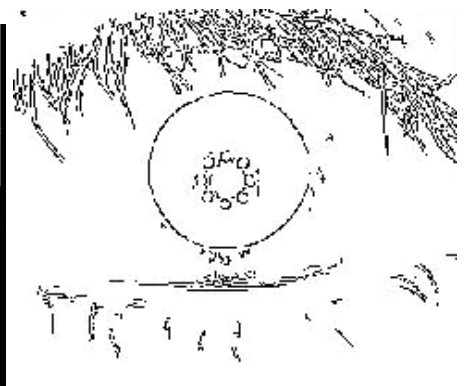

(c) and its Canny Edges

Fig. 6. (a) Original image (b) Half-Otsu (leftmost line) and Otsu values marked on the histogram of the Original Image.

also separate dark eyebrows too (Fig. 7(a)). But again here all is needed is an approximate radius plus or minus "delta". In other words small range for Hough circle search. That is $r_{\text {expected }} \pm \Delta_{r}$. This information is valuable in searching circles in the Hough space having radius in the range of $\left[r_{\text {expected }} \pm \Delta_{r}\right]$. Better and more efficient results from the Hough circle search can be obtained by reducing the radius range. It can be seen from the Fig. 7(a) and 7(b) that thresholding can give us good approximation for the pupil region.

\section{Algorithm 2}

1. Finding Otsu threshold value of the Original Image

2. Thresholding Original Image with the half of the Otsu value

3. Dilating and Eroding (Opening) Half-Otsu thresholded image Generate two instances of the resulting image: Inst1, Inst2

4. Performing Canny-Edge Detection on the thresholded image Inst1

5. Counting black pixels on the thresholded image Inst2 Estimating expected pupil radius ( $\left.\mathrm{r}_{\text {expected}}\right)$ on the thresholded image Inst2

6. Searching for Circles in Hough Space on the thresholded image Inst1 Searching circles with radius $=\left[\mathrm{r}_{\text {expected }} \pm \Delta \mathrm{r}\right]$

7. For Pupillary Boundary Finding the best match : The Hough circle that covers pupil edges best on the Inst1

8. Median Filtering Original Image Performing Gradient Search for limbic boundary on the Median Filtered Image

9. Extracting Normalized Image from the Original image

10. Background Subtraction and Bicubic scaling for the Normalized Image

11. Return Scaled and Enhanced Iris Band

The formula for the area of a disc is used for finding the approximate pupil radius. But before that one has to "clean" isolated black pixels and avoid counting them along with the black pixels that may remain on the borders. For the first problem, morphological operators with $3 \times 3$ rectangular structuring element, like in-place erode (where central pixel replaced by the minimum in the $3 \times 3$ neighbourhood) and in-place dilate (where central pixel replaced by the maximum in the $3 \times 3$ neighbourhood) (Step 3 in algorithm 2) on the thresholded binary image (white 0 and black 1 ) is used. Erosion is to clean isolated black pixels and dilation to "shrink" the regions that may remain in the pupil area due to specular highlight. Especially CASIA V3 database images acquired with camera having leds. The 
specular highlight in the CASIA V3 images makes simple thresholding methods unusable for finding pupillary boundary. For the second problem it helps to focus on the smaller central region on the image, assuming that the pupil is in the central region of the image, which is the case for CASIA databases used in the experiments (Step 5 in algorithm 2).

In the algorithm Hough circle searching function is used in finding limbic boundaries, which given radius range and several other parameters, returns the list of possible circles on the input image (Step 6 in algorithm 2). Thresholded (with half of the Otsu value) and morphologically processed image is given as an input to this function. In this case the function will be more focused on finding pupil boundary, which can be assumed as a circle (Fig. 7(c)). After Canny edge detection, function searches for the circles on the edge pixels. But one has to devise scoring method for finding the best matched circle for pupillary boundary. For this the circles returned by Hough circle search function are scored, based on the number of overlapped pixels with the Canny edge pixels. So the algorithm used separate copy of the Canny edge image from the same image that is given as an input to the Hough circle function (Step 4 in algorithm 2). For overlapping "relaxed" counting is used (Step 7 in our algorithm, see algorithm 2). Every pixels on the edge image that falls into the $3 \times 3$ neighbourhood of each circle pixels is counted (marked them to avoid double counting), instead of looking for "strict" overlapping. This has to be done since the pupillary boundary is not a perfect circle most of the time.
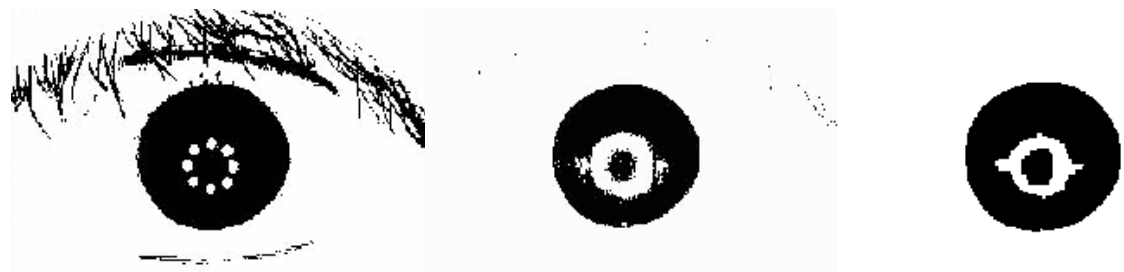

(a) Otsu thresh

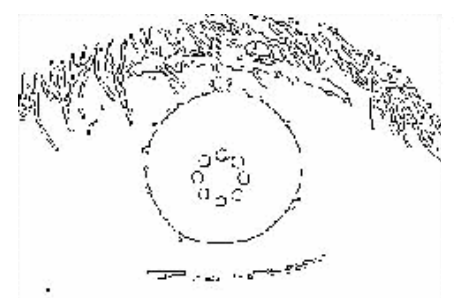

(d) Otsu thresh. (b) Half-Otsu thresh.

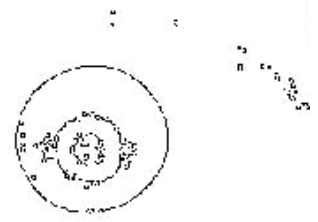

(e) Half-Otsu thresh. (c) After "Opening"

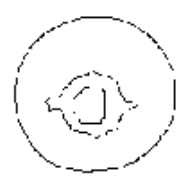

Fig. 7. 1st row: Otsu Thresholded image (a) and effect of "Opening" (c) (eroding and dilating black pixels) on Half-Otsu Thresholded image (b). 2nd row: Canny Edges of the; Otsu thresholded (d), Half-Otsu Thresholded (e), and after "Opening" (f). 
After finding pupillary boundary, using above given formulas (3) simple gradient search is utilized in both (a) Original Image (b) its Histogram (c) and its Canny Edges left and right directions, where differences of average grey level of the two consecutive windows (size is adjusted according to the image size and average grey level) observed on the median filtered image. Here the algorithm needs to find the point where the grey level starts increasing, in other words where the sclera region (generally whiter than the iris region) starts (Step 8 in our algorithm, see algorithm 2). Doing such a search on the original image can not give good results, as the histogram of the central (passing from the centre of the pupil) line (band in fact, 3-pixel wide) of the original image may contain little "spikes" (see Fig. 8). These "spikes" may be problem in finding the "real" place where the grey level starts increasing ( 0 is black and 255 is white). To overcome this difficulty median filtering is used, since median filtering preserves edges and smoothes boundaries without being computationally very expensive. The sample result of this search can be seen in Fig. 8, where the centre of the best matched circle is marked with little star and it is drawn in grey colour over the black pixels of the edges.

In this type of search method, crucial parameters are the width of the windows and the threshold value that will determine the actual place where the grey level increases. The window size is adjusted according to the image width and also the threshold is adjusted according to the average grey level of the image. The idea here is that, in bright images sclera can be found with threshold value greater than the ones where you have darker image. So smaller threshold values are used for darker images. The algorithm infact does not separate images into dark or light. To do better parameter calculations, small fuzzy-logic front-end is added to the algorithm which it was not included on the flow-chart (see algorithm 2). Basically we utilized the information acquired in the experiments. In the experiments it has been seen that setting global (for all images in the database) values for certain parameters, like thresholds, does not give good results. So in the algorithm the idea of adjusting them according to certain image characteristics is practised, as it is said above. The algorithm groups images according to their grey level averages. For example images having average grey level less than 100 can be classified as very dark images. Based on this the algorithm formed several groups based on the several ranges of the average grey level: [0..99], [100..140], [141..170], [171..200], [201..255]. Five groups are formed, which can be named as very dark, dark, middle, bright, very bright. For each group the algorithm adjusted certain parameters accordingly, also considering image size and expected radius of the pupil (which is calculated in step 5 of the algorithm, see algorithm 2). Finally in steps 9 and 10 (see algorithm 2) using "homogeneous rubber sheet" (Daugman,2004) segmented (see Fig. 8 for the iris band marked on the image) iris images are normalized (Fig. 8). The formulas used for normalization are described in next section. After background subtraction is made followed by bicubic scaling and histogram equalization. The results of these operations (steps 9-11) are described in section 2.3. Every iris band that are segmented scaled to the uniform size of 360x64.

\subsection{Iris normalization}

The irises captured from the different people have different sizes. The size of the irises from the same eye may change due to illumination variations, distance from the camera, or other factors. At the same time, the iris and the pupil are non concentric. These factors may affect the result of iris matching. In order to avoid these factors and achieve more accurate 

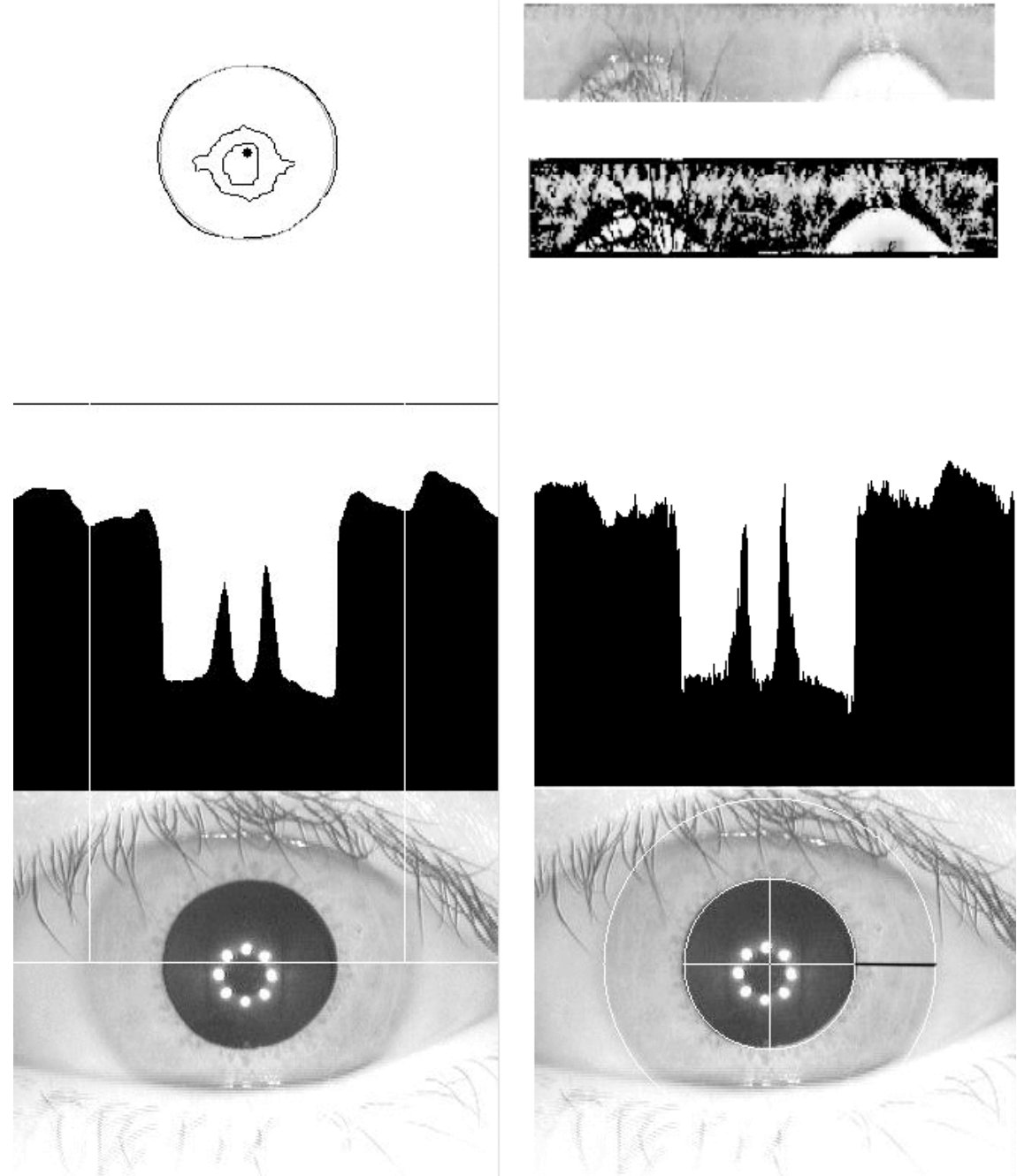

Fig. 8. Best-match Hough circle (left top) and detected limbic boundaries shown on the image (left bottom) and on the histogram of the central band (from the Median filtered image, left middle). Spikes on the histogram of the central (passing from the pupil centre) band from Original image (right middle). Detected iris band marked on the original image (right bottom). Normalized iris band (right uppermost). Scaled-Enhanced Normalized iris band (right second from the top).

recognition, the normalization of iris images is implemented. In normalization, the iris circular region is transformed to a rectangular region with a fixed size. With the boundaries detected, the iris region is normalized from Cartesian coordinates to polar representation. This operation is done using the following operation (Fig.9). 


$$
\begin{aligned}
& \theta \in[0,2 \pi], \quad r \in\left[R_{\mathrm{p}}, R_{L}(\theta)\right] \\
& x_{i}=x_{p}+r \cdot \cos (\theta) ; \quad y_{i}=y_{p}+r \cdot \sin (\theta)
\end{aligned}
$$

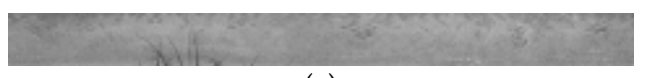

(a)

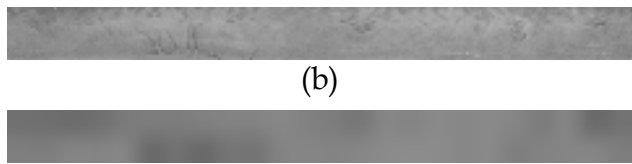

(c)

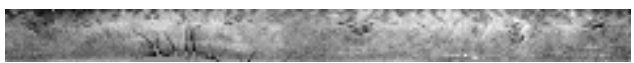

(d)

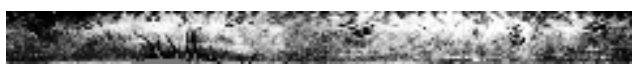

(e)

Fig. 9. a) Normalized image, b) Normalized image after removing eyelashes, c) Image of nonuniform background illumination, d) Image after subtracting background illumination, e) Enhanced image after histogram equalization.

Here $\left(x_{i}, y_{i}\right)$ is the point located between the coordinates of the papillary and limbic boundaries in the direction $\theta .\left(x_{p}, y_{p}\right)$ is the centre coordinate of the pupil, $R_{p}$ is the radius of the pupil, and $R_{L}(\theta)$ is the distance between centre of the pupil and the point of limbic boundary.

In the localization step, the eyelid detection is performed. The effect of eyelids is erased from the iris image using the linear Hough transform. After normalization (Fig. 9(a)), the effect of eyelashes is removed from the iris image (Fig. 9(b)). Analysis reveals that eyelashes are quite dark when compared with the rest of the eye image. For isolating eyelashes, a thresholding technique was used. To improve the contrast and brightness of image and obtain a well distributed texture image, an enhancement is applied. Received normalized image using averaging is resized. The mean of each $16 \times 16$ small block constitutes a coarse estimate of the background illumination. During enhancement, background illumination (Fig. 9(c)) is subtracted from the normalized image to compensate for a variety of lighting conditions. Then the lighting corrected image (Fig. 9(d)) is enhanced by histogram equalization. Fig. 9(e) demonstrates the preprocessing results of iris image. The texture characteristics of iris image are shown more clearly. Such preprocessing compensates for the nonuniform illumination and improves the contrast of the image.

Normalized iris provides important texture information. This spatial pattern of the iris is characterized by the frequency and orientation information that contains freckles, coronas, strips, furrows, crypts, and so on.

\section{Neural network based iris pattern classification}

\subsection{Neural Network based model}

In this paper, a Neural Network (NN) is used to recognise the iris patterns. In this approach, the normalized and enhanced iris image is represented by a two-dimensional array. This array contains the greyscale values of the texture of the iris pattern. These values are input 
signals for the neural network. Architecture of NN is given in Fig. 10. Two hidden layers are used in the NN. In this structure, $\mathrm{x}_{1}, \mathrm{x}_{2}, \ldots, \mathrm{x}_{\mathrm{m}}$ are greyscale values of input array that characterizes the iris texture information, $\mathrm{P}_{1}, \mathrm{P}_{2}, \ldots, \mathrm{P}_{\mathrm{n}}$ are output patterns that characterize the irises.

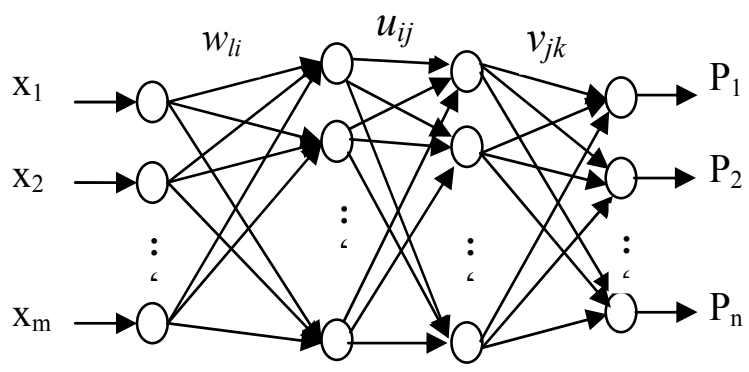

Fig. 10. Neural Network Architecture.

The k-th output of neural network is determined by the formula

$$
P_{k}=f_{k}\left(\sum_{j=1}^{h 2} v_{j k} \cdot f_{j}\left(\sum_{i=1}^{h 1} u_{i j} \cdot f_{i}\left(\sum_{l=1}^{m} w_{l i} x_{l}\right)\right)\right)
$$

where $v_{j k}$ are weights between the output and second hidden layers of network, $u_{i j}$ are weights between the hidden layers, $w_{i l}$ are weights between the input and first hidden layers, $f$ is the activation function that is used in neurons, $x_{l}$ is input signal. Here $k=1, . ., n$, $j=1, . ., h 2, i=1, . ., h 1, l=1, . ., m, m$ is number of neurons in input layer, $n$ is number of neurons in output layer, $h 1$ and $h 2$ are number of neurons in first and second hidden layers, correspondingly.

In formula (7) $P_{k}$ output signals of $\mathrm{NN}$ are determined as

$$
P_{k}=\frac{1}{1+e^{-\sum_{j=1}^{h 2} v_{j k} y_{j}}}
$$

where,

$$
y_{j}=\frac{1}{1+e^{-\sum_{i=1}^{h 1} u_{i j} y_{i}}} ; \quad y_{i}=\frac{1}{1+e^{-\sum_{l=1}^{m} w_{l i} x_{l}}}
$$

Here $y_{i}$ and $y_{j}$ are output signals of first and second hidden layers, respectively.

After activation of neural network, the training of the parameters of NN start. The trained network is then used for the iris recognition in online regime.

\subsection{Parameter learning}

In this paper, a gradient based learning algorithm with adaptive learning rate is adopted. This allows to guarantee convergence and speed up learning processes. In addition a momentum is used to speed up learning processes. 
At the beginning, the parameters of NN are generated randomly. The parameters $v_{j k}, u_{i j}$, and $w_{l i}$ of NN are weight coefficients of second, third and last layers, respectively. Here $k=1, . ., n$, $j=1, . ., h 2, i=1, . ., h 1, l=1, . ., m$. To generate NN recognition model, the training of the weight coefficients of $v_{j k}, u_{i j}$, and $w_{l i}$. s has been carried out.

During training the value of the following cost function is calculated.

$$
E=\frac{1}{2} \sum_{k=1}^{n}\left(P_{k}^{d}-P_{k}\right)^{2}
$$

Here $n$ is the number of output signals of the network and $P_{k}^{d}$ and $P_{k}$ are the desired and the current output values of the network, respectively. The parameters $v_{j k}, u_{i j}$, and $w_{l i}$ of neural network are adjusted by using the following formulas.

$$
\begin{aligned}
& w_{l i}(t+1)=w_{l i}(t)-\gamma \frac{\partial E}{\partial w_{l i}}+\lambda\left(w_{l i}(t)-w_{l i}(t-1)\right) \\
& u_{i j}(t+1)=u_{i j}(t)-\gamma \frac{\partial E}{\partial u_{i j}}+\lambda\left(u_{i j}(t)-u_{i j}(t-1)\right) \\
& v_{j k}(t+1)=v_{j k}(t)-\gamma \frac{\partial E}{\partial v_{j k}}+\lambda\left(v_{j k}(t)-v_{j k}(t-1)\right)
\end{aligned}
$$

Here $\gamma$ is the learning rate, and $\lambda$ is the momentum.

The derivatives in (11) are determined using the following formulas.

$$
\begin{aligned}
& \frac{\partial E}{\partial v_{j k}}=\frac{\partial E}{\partial P_{k}} \frac{\partial P_{k}}{\partial v_{j k}} ; \quad \frac{\partial E}{\partial u_{i j}}=\frac{\partial E}{\partial P_{k}} \frac{\partial P_{k}}{\partial y_{j}} \frac{\partial y_{j}}{\partial u_{i j}} ; \\
& \frac{\partial E}{\partial w_{l i}}=\frac{\partial E}{\partial P_{k}} \frac{\partial P_{k}}{\partial y_{j}} \frac{\partial y_{j}}{\partial y_{i}} \frac{\partial y_{i}}{\partial w_{l i}} ;
\end{aligned}
$$

The derivatives in (12) are determined as

$$
\begin{array}{ll}
\frac{\partial E}{\partial P_{k}}=\left(P_{k}-P_{k}^{d}\right) ; & \frac{\partial P_{k}}{\partial v_{j k}}=P_{k}\left(1-P_{k}\right) \cdot y_{j} \\
\frac{\partial P_{k}}{\partial y_{j}}=P_{k}\left(1-P_{k}\right) \cdot v_{j k} ; & \frac{\partial y_{j}}{\partial u_{i j}}=y_{j}\left(1-y_{j}\right) \cdot y_{i} \\
\frac{\partial y_{j}}{\partial y_{i}}=y_{j}\left(1-y_{j}\right) \cdot u_{i j} ; & \frac{\partial y_{i}}{\partial w_{l i}}=y_{i}\left(1-y_{i}\right) \cdot x_{l}
\end{array}
$$

Using equations (11-13), an update of the parameters of the neural network is carried out. One important problem in learning algorithms is convergence. The convergence of the gradient descent method depends on the selection of the initial values of the learning rate. Usually, this value is selected in the interval [0-1]. A large value of the learning rate may lead to unstable learning, a small value of the learning rate results in a slow learning speed. In this paper, an adaptive approach is used for updating these parameters. That is, the learning of the NN parameters is started with a small value of the learning rate $\gamma(t)$. During 
learning, $\gamma(t)$ is increased if the value of change of error $\Delta \mathrm{E}(\mathrm{t})=\mathrm{E}(\mathrm{t})-\mathrm{E}(\mathrm{t}+1)$ is positive, and decreased if negative. This strategy ensures a stable learning for the type- $2 \mathrm{NN}$, guarantees the convergence and speeds up the learning.

\section{Experimental results}

The CASIA iris image databases are used to evaluate the iris recognition algorithms. Currently this is one of largest iris database available in the public domain. The experiments are performed for two CASIA version 1 and CASIA version 3 iris databases. At first CASIA version 1 iris database is used. This image database contains 756 eye images from 108 different persons. Experiments are performed in two stages: iris segmentation and iris recognition. At first stage the above described black rectangle algorithm is applied for the localization of irises. The experiments were performed by using Matlab on Pentium IV PC. The average time for the detection of inner and outer circles of the iris images was $0.14 \mathrm{~s}$. The accuracy rate was $98.62 \%$. Also using the same conditions, the computer modelling of the iris localization is carried out by means of Hough transform and Canny edge detection realized by Masek and integrodifferential operator realized by Daugman. The average time for iris localization using Hough transform is obtained $85 \mathrm{sec}$, and $90 \mathrm{sec}$ using integrodifferential operator. Table 1 demonstrates the comparative results of different techniques used for iris localization. The results of Daugman method are difficult for comparison. If we use the algorithm which is given in (Daugman,2001; Daugman,2004) then the segmentation represents $57.7 \%$ of precision. If we take into account the improvements that were done by author then Daugman method presents $100 \%$ of precision. The experimental results have shown that the proposed iris localization rectangular area algorithm has better performance.

Second experiment was performed using algorithm based on Otsu thresholding and Hough circle. Experiments are performed using both CASIA1 version 1 and version 3 iris image databases. As mentioned CASIA version 1 image database contains 756 eye images from 108 different people, CASIA version 3 (Interval) database contains 2655 eye images from 249 different people. Experiments were performed on Intel ${ }^{\circledR}$ Quad Core $2.4 \mathrm{GHz}$ machine with openSUSE 11.0 Linux system and Intel C/C++ (V11.0.074) compilers with OpenCV (V1.0) and IPP (V6.0) libraries were used (all in 64 bit). IPP optimized mode is utilized for OpenCV, since IPP library can provide speedups up to $3.84 \mathrm{x}$ for multi-threading on a 4-core processor2. The implemented algorithm can extract iris band in about $0.19 \mathrm{sec}$.

This roughly equals to $5 \mathrm{fps}$ processing speed (for $320 \times 280$ resolution), with on line camera. The segmentation results for both databases are given in the Table 2 . We believe the proposed algorithm can be used for on line iris extraction with an acceptable real-time performance. After segmentation we have checked extracted iris images visually to see if there is any extracted image that can not be suitable input for any classification method. Total 7 images rejected after inspection, from the CASIA V3. In the case of CASIA V1 images we did not see any unsuccessful segmentation.

In second stage the iris pattern classification using NN is performed. For classification 50 different persons were selected from iris database. From each person two irises are used for training and two irises for testing. The detected irises after normalization and enhancement are scaled by using averaging. This help to reduce the size of neural network. Then the images are represented by matrices. These matrices are the input signal for the neural 


\begin{tabular}{|l|l|l|}
\hline Methodology & Accuracy rate & Average time \\
\hline Daugman [4] & $57.7 \%$ & $90 \mathrm{~s}$ \\
\hline Wildes [7] & $86.49 \%$ & $110 \mathrm{~s}$ \\
\hline Masek [9] & $83.92 \%$ & $85 \mathrm{~s}$ \\
\hline Proposed & $98.62 \%$ & $0.14 \mathrm{~s}$ \\
\hline
\end{tabular}

Table 1. Accuracy rate for iris segmentation.

\begin{tabular}{|l|l|l|l|l|}
\hline Version & Images & Segmented & Percent & Avg (sec) \\
\hline CASIA V1 & 756 & 748 & 98.94 & 0.18 \\
\hline CASIA V3 & 2655 & 2624 & 98.83 & 0.19 \\
\hline
\end{tabular}

Table 2. Segmentation Results for CASIA V1 (all) and V3 (CASIA-IrisV3-Interval).

network. The outputs of the neural network are classes of iris patterns. Each class characterizes the certain person's iris. Two hidden layers are used in neural network. The numbers of neurons in first and second hidden layers are 120 and 81, correspondingly. In the hidden layers the number of neurons is selected after several experiments by trial. The use of less number of neurons in hidden layer does not provide enough accuracy of network. To improve computational power of neural network and to decrease training error we increment number of neurons in hidden layer. Neural learning algorithm is applied in order to solve iris classification. From each set of iris images, two patterns are used for training and two patterns for testing. After training the remaining images are used for testing. The recognition rate of $\mathrm{NN}$ system was $99.25 \%$. The obtained recognition result is compared with the recognition results of other methods that utilize the same iris database. The results of this comparison are given in Table 3. As shown in the table, the identification result obtained using the neural network approach illustrates the success of its efficient use in iris recognition. But we need to note that future increasing the number of iris patterns decrease the recognition rate using $\mathrm{NN}$.

\begin{tabular}{|l|l|}
\hline Methodology & Accuracy rate \\
\hline Daugman [4] & $100 \%$ \\
\hline Boles [8] & $92.64 \%$ \\
\hline Li Ma [11] & $94.9 \%$ \\
\hline Avila [26] & $97.89 \%$ \\
\hline Neural Network & $99.25 \%$ \\
\hline
\end{tabular}

Table 3. The recognition performance of comparing with existing methods.

\section{Conclusion}

Personal identification using iris recognition is presented. Iris segmentation algorithms are proposed for accurate and fast detection of irise patterns. First one is based on detection of black rectangular patterns on purples. Second algorithm is based Otsu thresholding and Hough circle of iris patterns that can be efficiently applied to images containing specular 
highlights. The application of segmentation algorithms are demonstrated on CASIA version 1 an version 3 databases. Using black rectangle algorithm the average time for iris segmentation is obtained to be $0.14 \mathrm{sec}$ on Pentium IV PC using Matlab. Accuracy rate of iris segmentation $98.62 \%$ is achieved. For second algorithm the accuracy of iris segmentation for CASIA version 1 is 98.84 , for version $3-98.83 \%$. The located iris after pre-processing is represented by a data set. Using this data set as input signal the neural network is used to recognize the iris patterns. The recognition accuracy was $99.25 \%$. The obtaine results demonstrate the efficiency of proposed algorithms.

\section{References}

Jain A., Bolle R., and Kanti S. P. Biometrics: Personal Identification in a Networked Society. Kluwer, 1998.

Adler A. Physiology of Eye : Clinical Application. London, The C.V. Mosby Company, fourth edition, 1965.

Daugman J. Biometric Personal Identification System Based on Iris Analysis. US Patent no. 5291560, 1994.

Daugman J. Statistical richness of visual phase information: Update on recognizing persons by iris patterns. Int. Journal of Computer Vision, 2001.

Daugman J. Demodulation by complex-valued wavelets for stochastic pattern recognition. Int. Journal of Wavelets, Multiresolution and Information Processing, 2003.

Daugman J. How iris recognition works. IEEE Transactions on Circuits and Systems for Video Technology, 14(1):21 - 30, January 2004.

Wildes R. Iris recognition: An emerging biometric technology. Proc. of the IEEE, 85(9):1348 1363, September 1997.

Boles W. and Boashash B. A human identification technique using images of the iris and wavelet transform. IEEE Trans. on Signal Processing, 46(4):1185 - 1188, 1998.

Masek L. Recognition of Human Iris Patterns for Biometric Identification. BEng. Thesis. School of Computer Science and Software Engineering, The University of Western Australia, 2003.

Ma L., Tan T., Wang Y., and Zhang D. Personal identification based on iris texture analysis. IEEE Trans. Pattern Anal. Mach. Intelligence, 25(12):1519 - 1533, December 2003.

Ma L., Wang Y. H., and Tan T. N. Iris recognition based on multichannel gabor filtering. Proc. of the Fifth Asian Conference on Computer Vision, Australia, pages 279 - 283, 2002.

Tisse C., Martin L., Torres L. and Robert M. Person identification technique using human iris recognition. Proc. of Vision Interface, pages 294 - 299, 2002.

Kanag H. and Xu G. Iris recognition system. Journal of Circuit and Systems, 15(1):11 - 15, 2000.

Yuan W., Lin Z. and Xu L. A rapid iris location method based on the structure of human eyes. Proc. Of 27th IEEE Annual Conferemce Engineering in Medicine and Biology, Shanghai, China, September 1-4 2005.

Daugman J. New methods in iris recognition. IEEE Trans. Syst., Man, Cybern. B, Cybern., 37(5):1168 - 1176, October 2007.

Vatsa M., Singh R., and Noore A. Improving iris recognition performance using segmentation, quality enhancement, match score fusion, and indexing. IEEE Trans. 
on Systems, Man, and Cybernetics Part B: Cybernetics, 38(4):1021 - 1035, August 2008.

Zuo J. and Schmid N. An Automatic Algorithm for Evaluating the Precision of Iris Segmentation, IEEE Second Int. Conf. on Biometrics Theory, Applications and Systems (BTAS 08) Sep. 29 - Oct. 1, 2008.

Liu X., Bowyer K., and Flynn P. Experiments with an improved iris segmentation algorithm. Fourth IEEE Workshop on Automatic Identification Advanced Technologies, 17 18:118 - 123, 2005.

Cui J., Wang Y., Tan T., Ma L., and Sun Z. A fast and robust iris localization method based on texture segmentation. Proc. SPIE, 5404:401 - 408, 2004.

Abiyev R. and Altunkaya K. Neural Network Based Biometric Personel Identification with fast iris segmentation. Int. Journal of Control, Automation and Systems. Vol.7, No.1, 2009.

Abiyev R. and Altunkaya K. Iris recognition for biometric personal identification using neural networks. Lecture Notes in Computer Sciences, Springer-Verlag, 4669, 2007.

Abiyev R. and Altunkaya K. Personal Iris Recognition Using Neural Networks. International Journal of Security and its Applications, vol.2. No.2, April, 2008.

Abiyev R. and Kilic K. Adaptive Iris segmentation. Lecture Notes in Computer Sciences, Springer-Verlag, Berlin Heidelberg, CS press, 2009.

Daugman J. and Downing C. Recognizing iris texture by phase demodulation. IEEE Colloquium on Image Processing for Biometric Measurement, 2:1 - 8, 1994.

Miyazawa K., Ito K., Aoki T., Kobayashi K. and Nakajima H. An effective approach for iris recognition using phase-based image matching. IEEE Trans. on Pattern Analysis and Machine Intelligence, 30(10):1741 - 1756, October 2008.

Sanchez-Avila C. and Sanchez-Reillo R. Iris-based biometric recognition using dyadic wavelet transform. IEEE Aerospace and Electronic Systems Magazine, pages 3 - 6, 2002.

Noh S., Bae K. and Kim J. A novel method to extract features for iris recognition system. Proc. 4th Int. Conf. Audio and Video Based Biometric Person Authentication, pages $838-844,2003$.

Mallat S. Zero crossings of a wavelet transform. IEEE Trans. Inf. Theory, 37(4):1019 - 1033, April 1992.

Park C., Lee J., Smith M. and Park K. Iris based personal authentication using a normalized directional energy feature. Proc. 4th Int. Conf. Audio- and Video-Based Biometric Person Authentication, pages 224 - 232, 2003.

Lim S., Lee K., Byeon O. and Kim T. Efficient iris recognition through improvement of feature vector and classifier. ETRI J., 23(2):61 - 70, 2001.

Ma L., Tan T., Zhang D., and Wang Y. "Local intensity variation analysis for iris recognition", Pattern Recognition, vol. 37, no. 6,pp. 1287-1298, 2005.

Scotti F., "Computational intelligence techniques for reflections identification in iris biometric images", CIMSA 2007 - IEEE Int. Conf. on Computational Intelligence for Measurement Systems and Applications, Ostuni - Italy, 27-29 June 2007

Wang Y. and Han J. Q. Iris feature extraction using independent component analysis. Proc. 4th Int. Conf. Audio and Video Based Biometric Person Authentication, pages 838 844, 2003. 
Wang Y. and Han J. Q.. Iris recognition using independent component analysis. Proc. of the Fourth Int. Conf. on Machine Learning and Cybernetics, Guangzhou, 2005.

Otsu N. A threshold selection method from gray-level histograms. IEEE Trans. Sys., Man., Cyber., 9:62 - 66, 1979.

Sezgin M. and Sankur B. Survey over image thresholding techniques and quantitative performance evaluation. Journal of Electronic Imaging, 13(1):146 - 165, 2004.

Trier I. D. and Taxt T. Evaluation of binarization methods for document images. IEEE Trans. on Pattern Analysis and Machine Intelligence, 1995.

CASIA iris database. Institute of Automation, Chinese Academy of Sciences http://www.cbsr.ia.ac.cn/IrisDatabase.htm

Abiyev R.H. and Kilic K. An efficient Fractal Measure for Image Texture Recognition. International Conference on Soft Computing and Computing with Words in System Analysis, Decision and Control ICSCCW-2009,North Cyprus, Turkey, 2009 


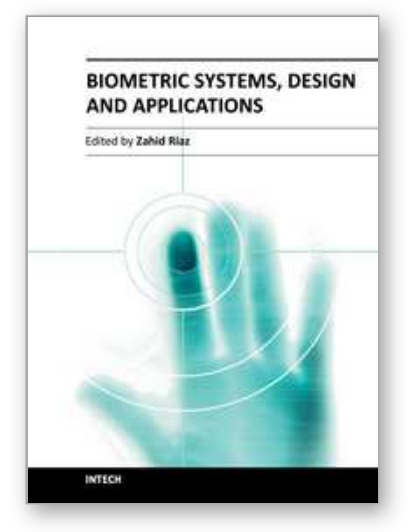

\author{
Biometric Systems, Design and Applications \\ Edited by Mr Zahid Riaz
}

ISBN 978-953-307-542-6

Hard cover, 262 pages

Publisher InTech

Published online 21, October, 2011

Published in print edition October, 2011

Biometric authentication has been widely used for access control and security systems over the past few years. The purpose of this book is to provide the readers with life cycle of different biometric authentication systems from their design and development to qualification and final application. The major systems discussed in this book include fingerprint identification, face recognition, iris segmentation and classification, signature verification and other miscellaneous systems which describe management policies of biometrics, reliability measures, pressure based typing and signature verification, bio-chemical systems and behavioral characteristics. In summary, this book provides the students and the researchers with different approaches to develop biometric authentication systems and at the same time includes state-of-the-art approaches in their design and development. The approaches have been thoroughly tested on standard databases and in real world applications.

\title{
How to reference
}

In order to correctly reference this scholarly work, feel free to copy and paste the following:

Rahib Hidayat Abiyev and Kemal Insan Kilic (2011). Robust Feature Extraction and Iris Recognition for Biometric Personal Identification, Biometric Systems, Design and Applications, Mr Zahid Riaz (Ed.), ISBN: 978953-307-542-6, InTech, Available from: http://www.intechopen.com/books/biometric-systems-design-andapplications/robust-feature-extraction-and-iris-recognition-for-biometric-personal-identification

\section{INTECH}

open science | open minds

\author{
InTech Europe \\ University Campus STeP Ri \\ Slavka Krautzeka 83/A \\ 51000 Rijeka, Croatia \\ Phone: +385 (51) 770447 \\ Fax: +385 (51) 686166 \\ www.intechopen.com
}

\author{
InTech China \\ Unit 405, Office Block, Hotel Equatorial Shanghai \\ No.65, Yan An Road (West), Shanghai, 200040, China \\ 中国上海市延安西路65号上海国际贵都大饭店办公楼 405 单元 \\ Phone: +86-21-62489820 \\ Fax: $+86-21-62489821$
}


(C) 2011 The Author(s). Licensee IntechOpen. This is an open access article distributed under the terms of the Creative Commons Attribution 3.0 License, which permits unrestricted use, distribution, and reproduction in any medium, provided the original work is properly cited. 